\title{
Boerhaave Syndrome in an Elderly Man
}

\author{
Maria João Ferreira Pinto ${ }^{1}$, Pedro Rodrigues ${ }^{1}$, Leonor Almeida ${ }^{2}$, Alexandra Leitão ${ }^{3}$, Luís Flores ${ }^{1}$, \\ André Gomes ${ }^{1}$, Gonçalo Rocha ${ }^{1}$, Fernando Friões ${ }^{1}$ \\ ${ }^{1}$ Internal Medicine Department, Centro Hospitalar de São João, Porto, Portugal \\ ${ }^{2}$ Pneumology Department, Centro Hospitalar de São João, Porto, Portugal \\ ${ }^{3}$ Internal Medicine Department, Hospital Santa Maria Maior, Barcelos, Portugal
}

How to cite this article: Pinto MJ, Rodrigues P, Almeida L, Leitão A, Flores L, Gomes A, Rocha G, Friões F. Boerhaave syndrome in an elderly man. EJCRIM 2018;5: doi:10.12890/2018_000944.

Conflicts of Interests: The Authors declare that there are no competing interests.

This article is licensed under a Commons Attribution Non-Commercial 4.0 License

\section{ABSTRACT}

Boerhaave syndrome is rare, has an non-specific clinical presentation and most commonly develops after persistent vomiting. Septic shock dominates the clinical picture as a result of extensive infection of the mediastinum and pleural and abdominal cavities. The current management of Boerhaave syndrome includes conservative, endoscopic and surgical treatments. The authors present the case of a 94-year-old man admitted to hospital with community-acquired pneumonia with mild respiratory insufficiency complicated by oesophageal perforation after an episode of vomiting and the development of a large left pleural effusion. An endoscopic approach with the placement of an oesophageal prosthesis was chosen given the advanced age of the patient. The hospital stay was complicated by pleural effusion infection requiring broad-spectrum antibiotics and prosthesis substitution. The patient was discharged after 60 days of hospitalization, without the need for oxygen supplementation, and scoring $80 \%$ on the Karnofsky Performance Status Scale. The increase in average life expectancy requires a case-by-case approach, where the benefits of invasive manoeuvres and likelihood of discharge are weighed against an acceptable quality of life, aiming to prevent futile medical treatment.

\section{LEARNING POINTS}

- Boerhaave syndrome is a complete rupture of the oesophageal wall secondary to a sudden increase in intraluminal oesophageal pressure, often in the lower third and left lateral position of the oesophagus.

- The management of Boerhaave syndrome depends on the time of diagnosis and clinical presentation and includes conservative, endoscopic and surgical approaches.

- Curative, aggressive approaches focused on the treatment of disease are often not appropriate for an aging population, hence the need for a case-by-case approach, where the benefits of invasive manoeuvres and likelihood of discharge are weighed against an acceptable quality of life, aiming to prevent futile medical treatment.

\section{KEYWORDS}

Oesophageal perforation; Boerhaave syndrome; pneumothorax; pleural effusion 


\section{CASE DESCRIPTION}

We present the case of a 94-year-old male patient with a known history of hypertension and dyslipidaemia, with no functional impairment, who scored $90 \%$ on the Karnofsky Performance Status Scale. He presented to the hospital with dyspnoea and cough, and was admitted with the diagnosis of community-acquired pneumonia with mild hypoxemic respiratory insufficiency (Fig. 1). Empirical antibiotic therapy with ceftriaxone and azithromycin was started.

On the second day of hospitalization, the patient experienced severe vomiting after dinner and developed worsening dyspnoea and marked bronchospasm later that day. On physical examination there were rhonchi and wheezing, and diminished breath sounds on the left. The patient showed aggravated hypoxaemic respiratory insufficiency and was transferred to an intermediate care unit.

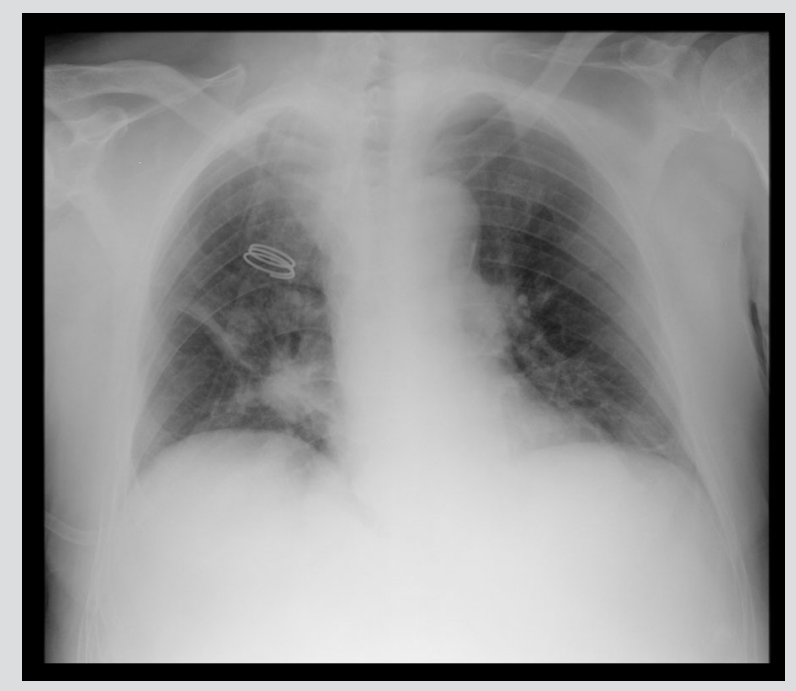

Figure 1. Chest $X$-ray showing pneumonia in the right middle lobe

\section{METHODS AND PROCEDURES}

Chest X-ray revealed a homogeneous opacity in the left hemithorax and a chest computed tomography (CT) scan showed a large left pleural effusion and a small anterior pneumothorax; the right lung was clear (Fig. 2).

A thoracocentesis revealed pleural fluid consistent with an exsudate: $\mathrm{pH} 6.6$, amylase 19,734 U/I (serum amylase $98 \mathrm{U} / \mathrm{I}$ ) and glucose <10 $\mathrm{mg} / \mathrm{dl}$. A chest tube was placed and a thoracoabdominal CT scan with oral contrast confirmed an oesophagopleural fistula, providing the diagnosis of Boerhaave syndrome (Fig. 3).

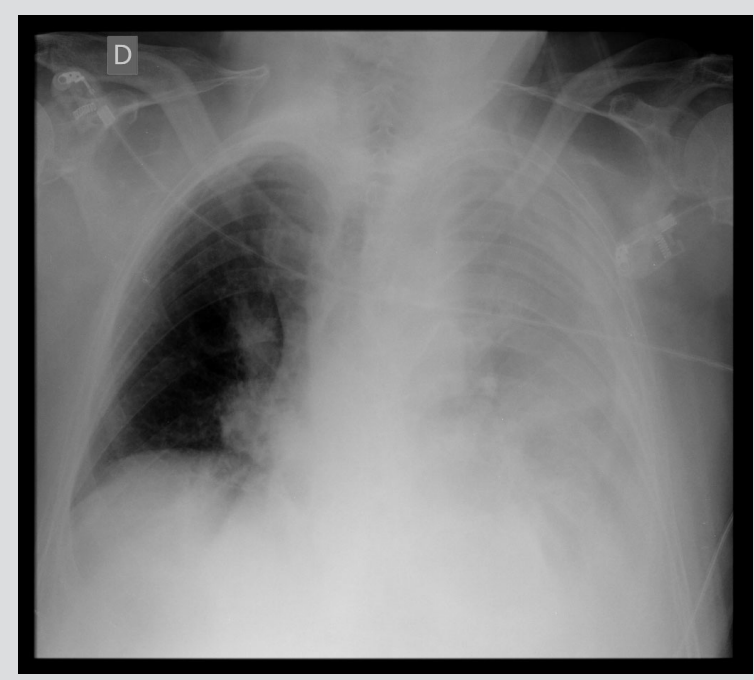

Figure 2. Chest $X$-ray showing homogeneous opacity in the left hemithorax 


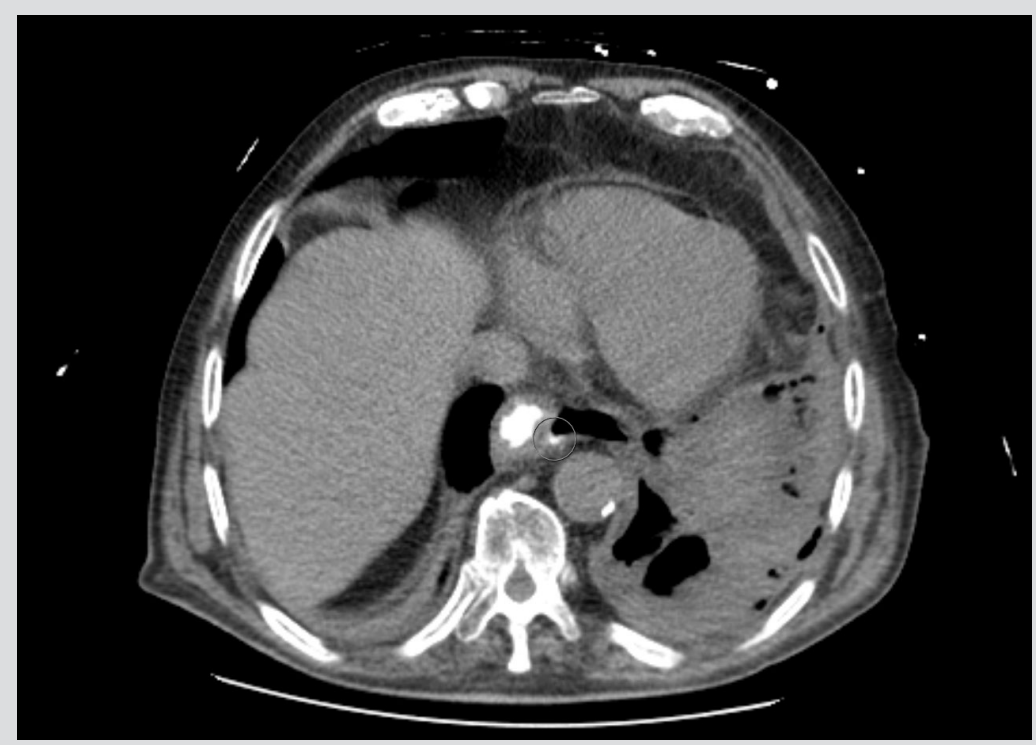

Figure 3. Thoracoabdominal CT with oral contrast showing contrast extravasation in the distal oesophagus

After stabilization, an endoscopy was performed and a $2 \mathrm{~cm}$ longitudinal ulceration in the distal oesophagus was revealed. A self-expanding metal prosthesis was placed.

Hospitalization was complicated by pleural effusion infection requiring broad-spectrum antibiotic therapy (piperacillin-tazobactam, vancomycin and anidulafungin) and prosthesis replacement due to insufficient coaptation of the upper segment of the previous prosthesis. The patient was discharged after 60 days of hospitalization, with no respiratory insufficiency, and scoring $80 \%$ on the Karnofsky Performance Status Scale.

\section{DISCUSSION}

Boerhaave syndrome is defined as a complete rupture of the oesophageal wall secondary to a sudden increase in intraluminal oesophageal pressure, most commonly after persistent vomiting. Oesophageal ruptures usually occur during diagnostic or therapeutic endoscopic procedures, trauma or various oesophageal diseases. Spontaneous rupture is uncommon, and represents $15 \%$ of all oesophageal ruptures ${ }^{[1]}$. It is more frequent in the lower third of the oesophagus and in the left lateral position, which might be due to an anatomic weakness in this $\operatorname{area}^{[2]}$.

Clinical manifestations of oesophageal rupture are often non-specific, depending on the location of the rupture and the length of time between its development and examination. Signs and symptoms such as chest pain, dyspnoea and fever are common and mimic those of other diseases. The clinical picture may be dominated by signs and symptoms of sepsis, with the patient rapidly deteriorating as result of extensive infection of the mediastinum and pleural and abdominal cavities ${ }^{[3]}$.

Laboratory findings are not specific and radiographs may show signs of pneumomediastinum, pneumothorax or hydropneumothorax. If pleural effusion is present, diagnostic thoracentesis can reveal the presence of food remnants, increased amylase and $\mathrm{pH}$ below 6 . The diagnosis is established by contrast oesophagram or a CT scan when there is evidence of contrast leakage from the oesophageal lumen ${ }^{[3]}$.

Current management of Boerhaave syndrome includes conservative, endoscopic and surgical treatments. If the diagnosis is made within the first 48 hours, the chosen approach depends on clinical stability, with surgical treatment being favoured when sepsis settles, whereas the endoscopic approach is preferred when there are no signs of sepsis and/or minimal contamination of the pleural cavity and mediastinum. Conservative management consisting of antibiotic treatment and percutaneous drainage of abscesses can be proposed to patients with delayed diagnosis, no signs of sepsis, and good tolerance of pleural contamination, despite the scarce available literature on this approach. If the endoscopic or conservative approach fails, patients are usually reconsidered for surgical management. The estimated global mortality rate of patients with Boerhaave syndrome is $20-40 \%{ }^{[2,4]}$.

In this case, the patient developed a large pleural effusion with an associated small pneumothorax, but had no signs of septic shock or a marked systemic inflammatory response, the latter explained perhaps by the patient's age, time of diagnosis, prompt treatment with timely drainage of pleural effusion, in a subject already being treated with antibiotics. The endoscopic approach was adopted, and despite the long hospital stay and the need for prosthesis replacement, the outcome was successful. 


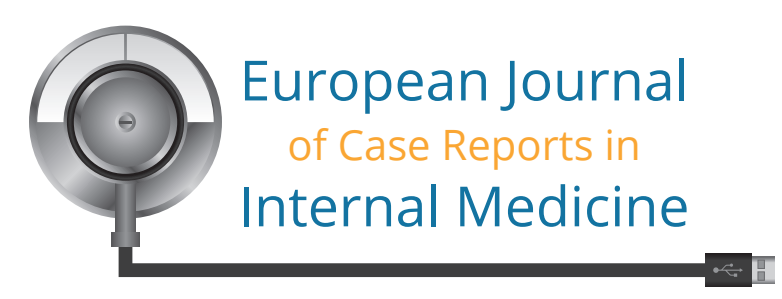

The increase in the average life expectancy requires a case-by-case approach, where the benefits of invasive manoeuvres and likelihood of discharge are weighed against an acceptable quality of life. Therefore, a multidisciplinary team consisting of surgeons, pneumologists, gastroenterologists, rehabilitation physicians and internists (pivotal in this case) is needed, guiding treatment throughout hospitalization, ensuring the special needs and requirements of the elderly are met, and continuously re-evaluating medical decisions to prevent futile medical treatment.

\section{REFERENCES}

1. Brinster CJ, Singhal S, Lee L, Marshall MB, Kaiser LR, Kucharczuk JC. Evolving options in the management of esophageal perforation. Ann Thorac Surg 2004;77:1475-1483.

2. de Schipper JP, Pull ter Gunne AF, Oostvogel HJ, van Laarhoven CJ. Spontaneous rupture of the oesophagus: Boerhaave's syndrome in 2008. Literature review and treatment algorithm. Dig Surg 2009;26:1-6.

3. Rokicki M, Rokicki W, Rydel M. Boerhaave's syndrome-over 290 yrs of surgical experiences. Epidemiology, pathophysiology, diagnosis. Pol Przegl Chir 2016;88:359-364.

4. Rokicki M, Rokicki W, Rydel M. Boerhaave's syndrome - over 290 yrs of surgical experiences. Surgical, endoscopic and conservative treatment. Pol Przegl Chir 2016;88:365372. 